measured from the cross-index line. The technique using the parallel cursor would be to set the range strobe to the distance-off (D.O.) and the cursor to the course to make good; the ship can then be conned so that the required point of land travels down the D.O. line. At about half to one mile from the new course (depending on speed), the cursor can be set to the new course to make

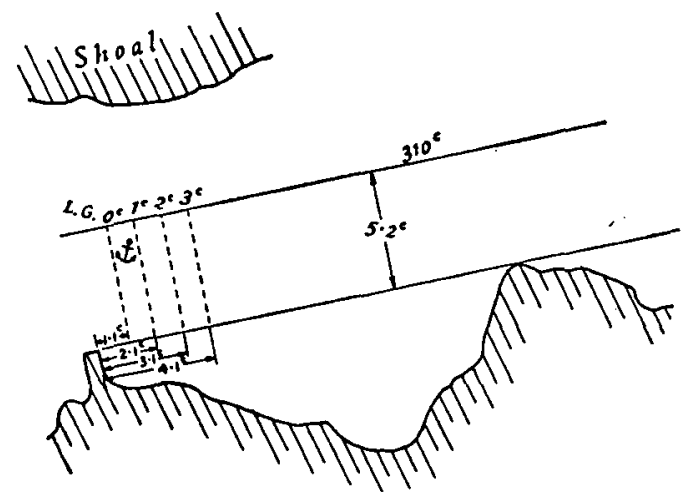

Fig. 4 good and the strobe to the new D.O. Dividers set to either the wheel-over distance off or the parallel distance to new course, can then be used to lay off the wheel-over position on the mask. For an anchorage with no ahead bearings, it is advisable to draw the distances to go along the D.O. line on the mask measured from the cross index line. As the ranging point travels down the line the distance to go can be seen at a glance.

Similar techniques can be used to great advantage when manœuvring in darkness or poor visibility. For manœuvring it is convenient to have a relativespeed scale, graduated for 3 or 6 minutes on the 10 - and 3-mile range scales, fixed to the PPI. The $3 \mathrm{~min}$. scale will give an approximate plot and the $6 \mathrm{~min}$. scale an accurate one. A relative turning data diagram will be valuable for warships who require to use turning data when working in close company.

These notes refer only to the use of a gyro-stabilized PPI; the techniques described should not be used with an unstabilized picture.

\title{
The Region of Collision
}

$$
\text { from W. T. Slater }
$$

Captain Wylie's paper on 'The Region of Collision'* raises some interesting points but if one accepts the view that graphing of some sort is not wholly 'academic' the writer feels that there is an alternative graph which Captain Wylie has not mentioned and is worth considering.

As Captain Wylie has shown, the time-distance plot gives very little useful information except that it does keep prominently in mind the distance between the two vessels. The time-bearing plot is much more informative as to the element of danger present in the situation but if the time-bearing is the only plot kept then the fact that it shows no distance between the vessels is a serious disadvantage. The writer suggests that it might be worthwhile considering the advantages of plotting change of bearing against distance between the vessels, especially if this is done on a graph having precomputed curves for various

* Wylie, F. J. (1956). The region of collision. This Journal, 9, 16r. 
minimum separations between the vessels. The sort of graph obtained is illustrated in Fig. I which shows a family of curves for vessels maintaining steady courses and passing at minimum separations of $\frac{1}{2}$, I and $\mathrm{I} \frac{1}{2}$ miles, curves $A, B$ and $C$ respectively, on the assumption that plotting is begun from the point where the vessels are four miles apart.

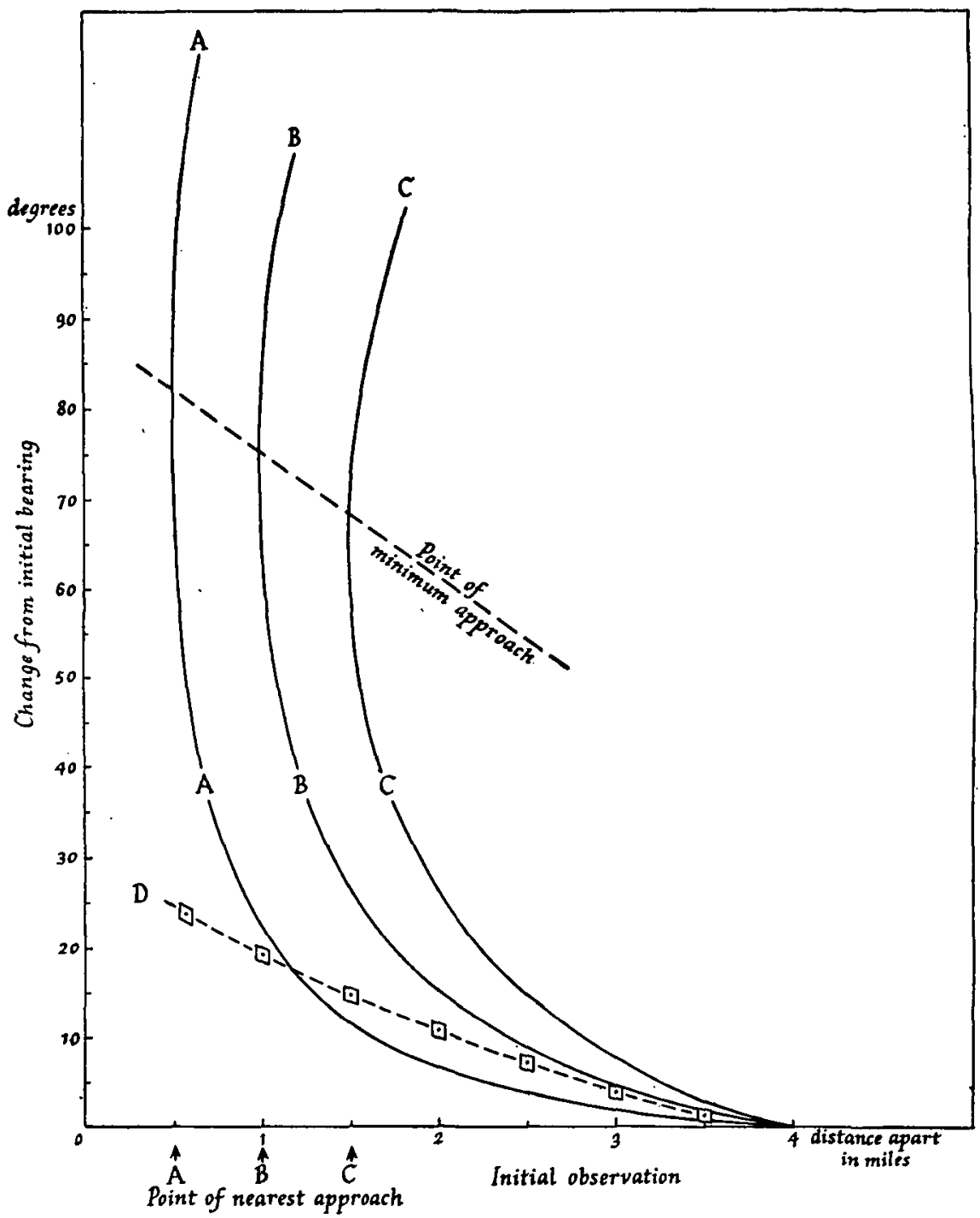

Fig. 1

For safe passing the actual graph of change of bearing against distance must closely follow the general trend of the precomputed curves. If the actual graph does not curve away in the manner of the precomputed curves and especially if the graph moves to the left of the precomputed curve for the minimum desired separation, then a dangerous situation is arising. In Fig. I curve $D$ is the graph 
approximately equivalent to the case suggested by Dr. Sutton and quoted in Captain Wylie's paper. Although the bearings are increasing, by the time separation is down to two miles it can be seen that the minimum distance apart at passing will be well below one mile; when separation is down to $\mathrm{I} \frac{1}{2}$ miles it is quite obvious that the graph is a long way from following the general trend of the family of curves and that separation at passing will be under half a mile. By the time the separation is down to one mile it is very obvious that a really dangerous situation has arisen and that the two vessels will rapidly arrive at a point where there is no distance between them.

Although the precomputed curves shown in Fig. I have been assumed to begin at four miles separation between the two vessels, the same figure can very simply be used for other initial separations merely by changing the scale of the distance ordinate. For instance if the observations are begun at eight miles apart the distance scale shown in Fig. I can be doubled and, in this case, curve $A$ gives the graph for a minimum separation of one mile, $B$ two miles and $C$ three miles. Similarly the figure could, if desired, be used for the case of observations beginning at twelve miles, when curve $A$ would indicate a minimum separation of $1 \frac{1}{2}$ miles, curve $B$ three miles and so on.

The observed information used to produce the graph in Fig. I is, of course, exactly the same as that to prepare a plot of course and distance on the face of the radar tube; but in the present case it is expressed in rectangular coordinates and, in the writer's view, results in a much greater sensitivity to departures from safe trends, partly as a result of the presence of the precomputed lines.

The graphs are, of course, independent of the relative speed of approach and, in fact, tables for these graphs could be computed somewhat on the lines of the table given by Captain Wylie in his paper; an example is given below showing the table for commencement of observations at eight miles separation. If the changes in bearing as distance decreases indicate that the bearings. are moving from one column to a column on the left then a state of danger would be indicated. Movement towards columns on the right would, of course, show an improving situation. By multiplying by a factor the figures for distance, i.e. miles apart and minimum approach, the same table can be used to check observations

\section{CHANGES OF BEARING DURING APPROACH CALCULATED FROM} INITIAL BEARING TAKEN AT 8 MILES

\begin{tabular}{cccc}
\hline $\begin{array}{c}\text { Miles } \\
\text { apart }\end{array}$ & \multicolumn{3}{c}{$\begin{array}{c}\text { Changes of bearing for minimum } \\
\text { approach of }\end{array}$} \\
8 & I n.m. & 2 n.m. & 3 n.m. \\
7 & - & - & - \\
6 & 1.0 & 2.1 & 3.4 \\
5 & 2.4 & 5.0 & 8.0 \\
4 & 4.3 & 9.1 & 15.0 \\
3 & 7.3 & 15.5 & 26.6 \\
2.5 & 12.3 & 27.3 & 68.0 \\
2 & 16.4 & 38.6 & - \\
1.5 & 22.8 & 75.5 & - \\
1 & 34.6 & - & - \\
\hline
\end{tabular}


from initial bearings taken at other distances apart just as the graphs can be used with various distance scales.

So far the use of the chart has been considered only for the purpose of estimating the closeness of approach of two vessels on steady courses, i.e. it has been assumed that the bearing is either steadily increasing or steadily decreasing and it is then necessary only to plot the amount of change from the initial bearing and its sign can be ignored. It appears, however, that the use of the

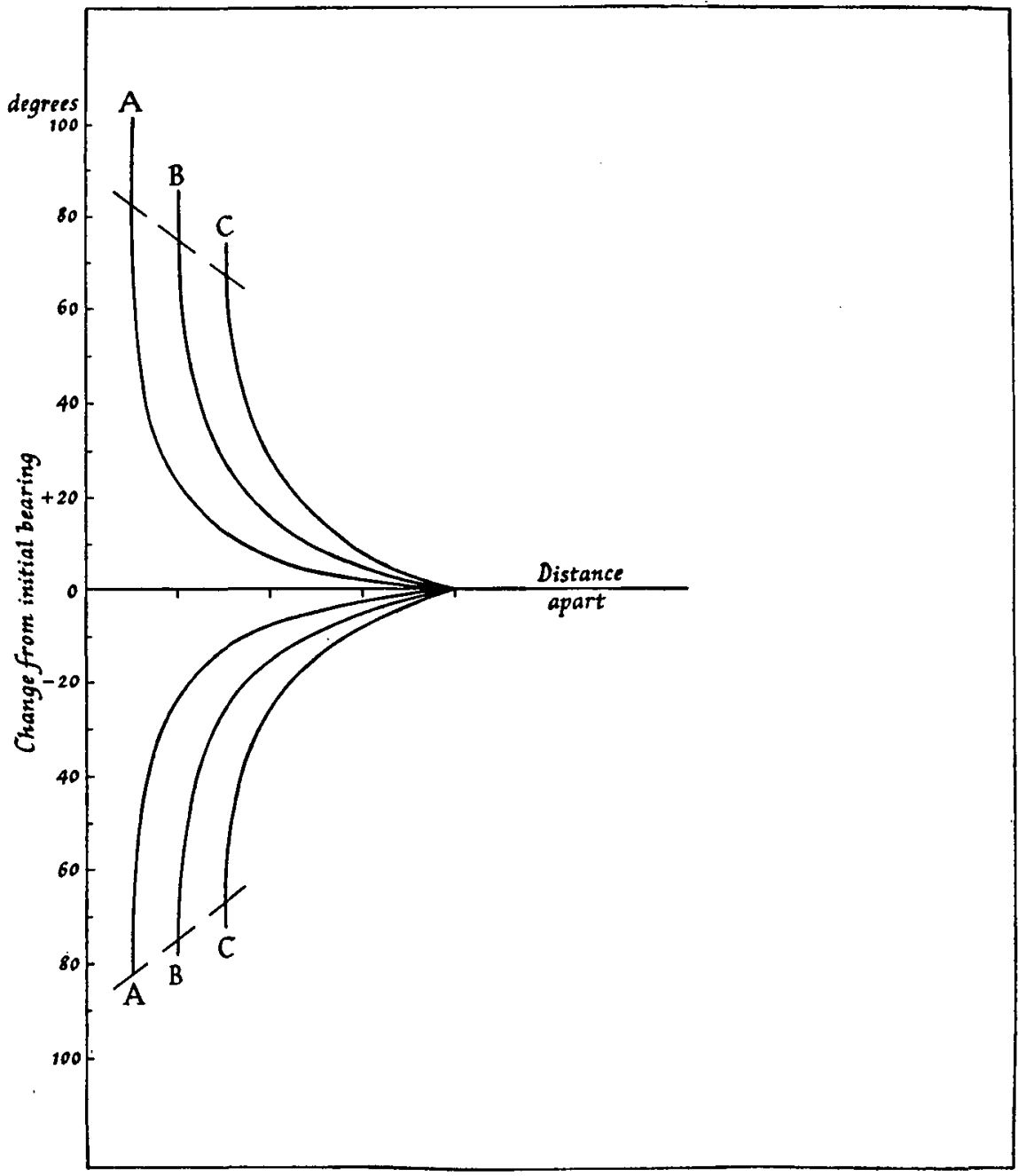

Fig. 2

chart might be extended to cover the case where the approaching ship changes course in such a way that the change of value of the bearings reverses. To avoid the slightest possibility of misunderstanding in such a case it may be desirable to consider a standard graph similar to Fig. 2 where positive changes in bearing are plotted upwards and the negative changes downwards. The upper part of Fig. 2 is, of course, the same as Fig. I whereas the lower part is a mirror image. 
In Fig. 3 is shown by curve $E$ the graph of change of bearings v. distance for a vessel which originally would have passed mile ahead of the graphing vessel hut when four miles away turns to starboard so as to pass astern at a minimum approach of I $\frac{3}{4}$ miles. Curve $E$ shows quite clearly:

(a) That on its original track the vessel would have passed within something under a mile of the plotting vessel.

(b) The sudden change when the vessels are four miles apart.

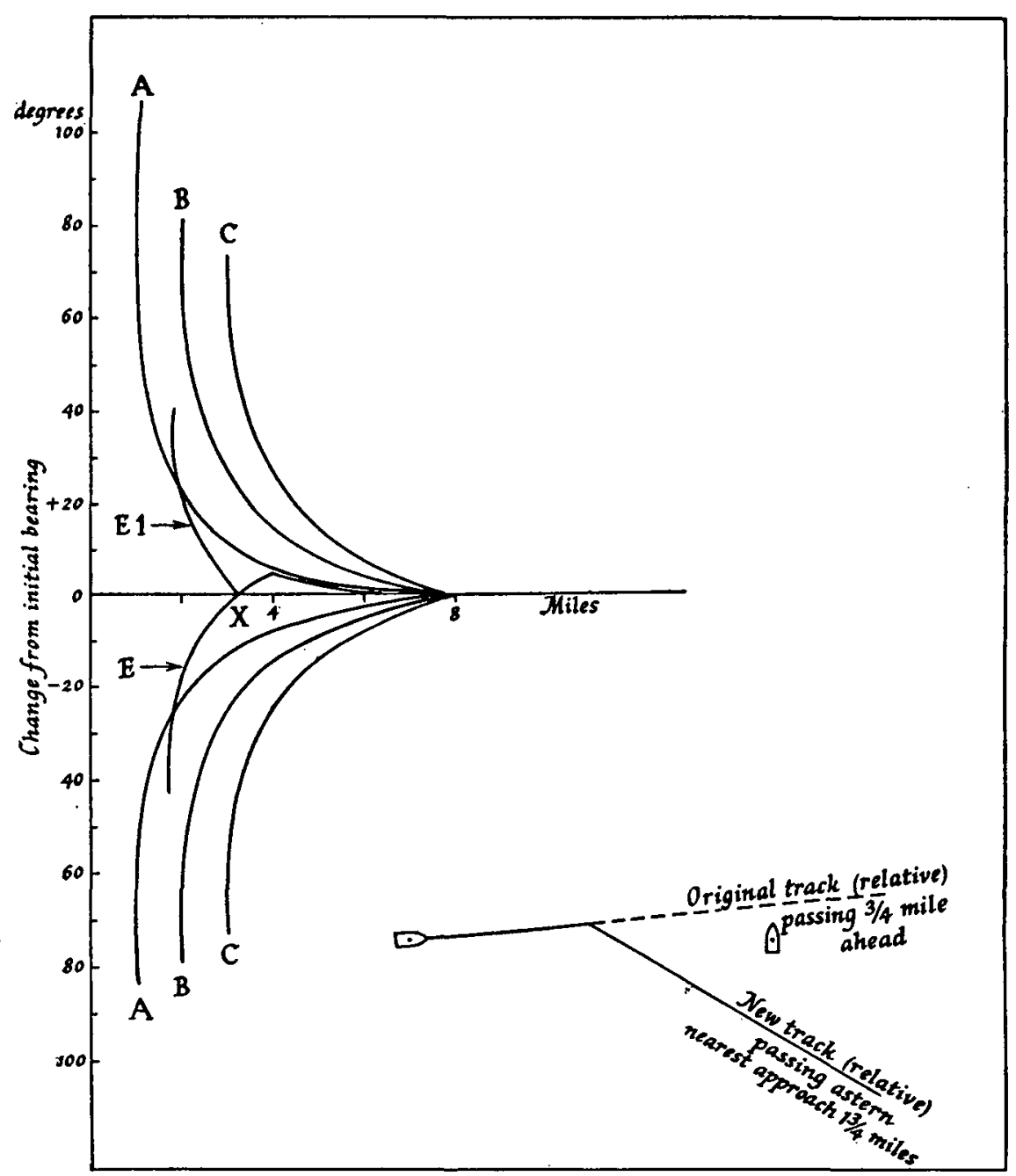

Fig. 3

As is to be expected, the vessel comes back to its original bearing and, therefore, crosses the zero line at $\mathrm{X}$, continuing downwards as the bearing continues to decrease. However, the way in which the curve continues very quickly indicates that the vessel is keeping its distance and is tending to pass clear with a separation of about 19 miles. 
Using Fig. I alone and ignoring the sign of the bearing change the curve $E^{\prime}$ would be obtained, which again is a mirror image of $E$ and shows almost equally clearly the satisfactory trend of events. It is possible, however, that the reversal of direction of curve $E^{\prime}$ at the point X might be misinterpreted and it is for this reason that the double graph may be considered preferable.

The curves in the figures are quite easy to compute but the writer will willingly send anyone interested to try the method, copies of Fig. I, of size approximately $8 \times 10 \mathrm{in}$., if the user is prepared to give a brief report on his results."

\section{Captain F.J. Wylie comments:}

Mr. Slater's curves present an interesting alternative to the use of tables. I would say at once that the principal shortcoming of both my suggested table and his curves is that they do no more than warn that all is not well; they do not give information which would help choice of manœuvring action. The table and curves are, of course, built on exactly the same data, the main difference between $\mathrm{Mr}$. Slater and myself being that his presentation deals with actual distances, whereas mine uses factors relative to the initial range used. It appears to me impossible to construct either a single table or a single set of curves to cover a variety of initial ranges except on the factoral basis I have used. If one uses that, one must accept a little simple arithmetic. It seems possible to make Mr. Slater's curves comprehensive only by similar or perhaps less simple arithmetic or by waiting for the range to close to a convenient (arithmetically) figure.

Basically, I prefer curves to tables, but in this case I am not yet convinced and will be most interested to hear more comment.

\section{D.f. Loop Errors due to Heel}

\section{from Francis Chichester}

YACHT and other small-boat navigators must often wonder what error in a d.f. loop reading on a radiobeacon may be due to heel. The error is in fact quite small, the maximum for a $25^{\circ}$ angle of heel being $2: 8$ when the bearing is quadrantal to the boat's heading, i.e. when the relative bearing is $45^{\circ}, 135^{\circ}$, $225^{\circ}$ or $315^{\circ}$. When the relative bearing is $0,90^{\circ}, 180^{\circ}$ or $270^{\circ}$, i.e. when the beacon is ahead or astern or abeam, the error is nil.

Table I gives corrections to the nearest $\frac{1}{2}$ degree, to be applied to the relative bearing of a beacon for $15^{\circ}$ and $25^{\circ}$ of heel. It makes no difference whether the boat is heeled towards or away from the beacon.

As the heel increases the complete arc of silence of the loop will disappear because the bottom of the tilted loop will be nearer to the beacon than the top of the loop and some signal will result. In practice this makes little difference to the observing; the loop operator will probably write it off as bad conditions because of the lively movement likely to accompany $25^{\circ}$ of heel in a seaway.

* The Institute will gladly forward correspondence to Mr. Slater. 